\title{
Dynamique sédimentaire d'un petit prodelta méditerranéen : le système Têt-littoral roussillonnais
}

\author{
François Bourrin ${ }^{1}$, Xavier Durrieu de Madron ${ }^{2}$, Centre de Formation et \\ de Recherche sur l'Environnement Marin (CEFREM), Université de \\ Perpignan, UMR 5110, 52 avenue Paul Alduy 66860 Perpignan \\ fbourrin@univ-perp.fr \\ ${ }^{1}$ doctorant, ${ }^{2}$ chargé de recherche CNRS
}

\begin{abstract}
Résumé
Le suivi sur une année entière des courants, de la concentration particulaire estimée par méthode acoustique et du niveau altimétrique du sédiment a été entrepris afin d'étudier la variabilité du transport sédimentaire sur le fond $(28 \mathrm{~m})$ d'un petit prodelta méditerranéen caractérisé par des apports continentaux discontinus et dominé par la houle. Les résultats montrent une érosion lente et continue pluri-centimétrique de la zone prodeltaïque tout au long de la période étudiée. Les flux de MES indiquent que le matériel érodé et remis en suspension, est dirigé vers le sud-est par les courants et alimenterait la vasière située au milieu du plateau continental avant d'être exporté hors du plateau. Les petits prodeltas méditerranéens agiraient ainsi comme des zones de «bypass » entre le continent et le bord de la marge continentale.
\end{abstract}

\begin{abstract}
$\underline{\text { Abstract }}$
A one-year data record of currents, near-bed suspended-sediment concentrations estimated by ADCP and altimetric variations of the seabed were analysed to evaluate the variability of suspended-sediment transport on the bottom $(28 \mathrm{~m})$ of a small event-dominated prodelta in NW Mediterranean characterized by flashflood events. Results of this analysis show a slow and continuous erosion of several centimetres of the prodelta area. Near-bed suspended-sediment fluxes indicate that eroded material and suspended material were advected southeastward and would feed the mid shelf bud-belt before being exported out of the margin. Small Mediterranean prodeltas acts as «bypass » areas between land and the exits of the continental margin.
\end{abstract}

Mots-clés: prodelta, Méditerranée, MES, hydrodynamique, flux, sédiments.

\section{Introduction}

Le Golfe du Lion est un bel exemple de marge continentale à construction deltaïque. Il est influencé à la fois par les apports constants de matériel continental du Rhône, un des plus grands fleuves méditerranéens, et par une multitude de

DOI: 10.5150/jngcgc.2006.035-B $\quad$ (disponible en ligne - http://www.paralia.fr - available online) 
fleuves côtiers à caractère impulsionnel; dont les principaux sont du nord au sud : le Vidourle, le Lez, l'Hérault, l'Orb, l'Aude, l'Agly, la Têt et le Tech (Figure 1a). En face de chaque distributaire, dans la zone littorale, il a été mis en évidence une zone de dépôt préférentielle plus ou moins temporaire de matériel fin d'origine continentale ${ }^{[1]}{ }^{[2]}$. Ces zones côtières, communément appelées prodeltas sont généralement situées sur des fonds allant de 25 à $30 \mathrm{~m}$ et constituées par des dépôts vaseux. Ceux-ci forment l'enregistrement sédimentaire des évènements météo-marins agissant sur l'ensemble bassin versant, fleuve, prodelta.

Le système Têt - Littoral du Roussillon, a été choisi car il se situe à la sortie du Golfe du Lion en aval des apports fluviatiles de tous les autres systèmes côtiers. Il est composé du fleuve Têt qui est un exemple de fleuve côtier méditerranéen de type torrentiel, et de son prodelta qui est une zone de dépôt temporaire du matériel fluviatile. L'essentiel des apports de matériel continental du fleuve Têt à la zone côtière s'effectue lors des courts évènements de crues ${ }^{[3]}$. Les mécanismes haute fréquence d'apport, de dépôt temporaire dans la zone prodeltaïque et d'érosion ont été étudiés lors d'évènements extrêmes de crue et tempête au niveau de ce système ${ }^{[4]}$. Il reste cependant à étudier l'impact de ces mécanismes sur une période plus longue (une année entière) afin de faire un bilan hydro-sédimentaire sur un petit prodelta méditerranéen caractérisé par des apports continentaux discontinus et dominé par les tempêtes. Alors que la dérive littorale, résultante du transport de sables lors des évènements de tempête de sud-est ${ }^{[5]}$, est dirigée du sud vers le nord sur le littoral roussillonnais, comment se comporte le matériel fin? Et sachant que les prodeltas agissent comme des zones de dépôt temporaire du matériel d'origine continentale, quel est le devenir final de celui-ci ?

\section{Environnement géologique et sédimentaire}

La couverture sédimentaire du Golfe du Lion se caractérise par plusieurs faciès qui sont répartis principalement suivant l'intensité des forçages hydrodynamiques depuis la côte jusqu'au bord du plateau continental (Figure 1a). Aux sables côtiers succède un mélange de silts et vases puis la vasière médiane vers $50-60 \mathrm{~m}$ de profondeur, et enfin les sables reliques du large caractérisant un ancien cordon littoral. L'épaisseur des sédiments vaseux holocènes est également représentée et montre l'importance des dépôts fins au nord-est du plateau en relation avec l'importance des apports rhodaniens et la circulation moyenne du courant d'est en ouest sur le plateau.

La dispersion des apports continentaux via les panaches fluviaux est contrainte par la circulation moyenne sur le plateau qui est principalement induite par le vent. Les vents dominants sont les vents continentaux provenant du nord $(70 \% \mathrm{du}$ temps en moyenne), la Tramontane et le Mistral, et induisent une circulation généralement cyclonique au niveau du plateau roussillonnais ${ }^{[6]}$. Les vents marins provenant du sud-est (30\% du temps) sont à l'origine des tempêtes et des crues qui sont provoquées par les précipitations sur les reliefs côtiers. Ces vents produisent également une circulation cyclonique sur le plateau roussillonnais qui induit la formation d'un long fleuve turbide côtier lors des évènements de sud-est. 


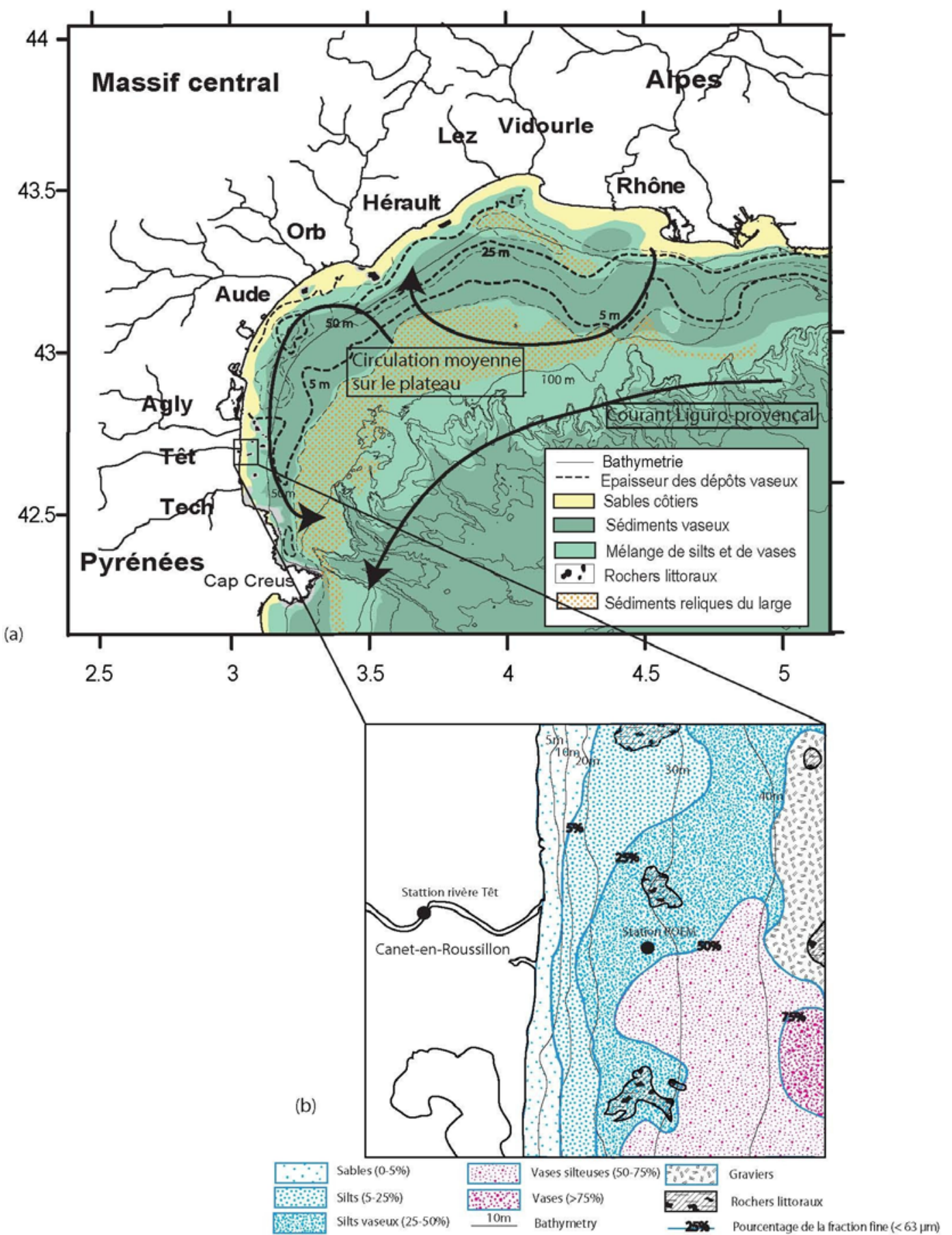

Figure 1 : a, Carte sédimentologique du Golfe du Lion (adaptée de Aloisi et al., $1973^{[7]}$ ) et b, carte sédimentologique détaillée du prodelta du fleuve Têt (carte géologique de Perpignan $1 / 50000^{\mathrm{e}}$ modifiée). La circulation moyenne sur le plateau et le courant Liguro-provençal au large sont également représentés.

La carte sédimentologique détaillée du prodelta du fleuve Têt (Figure 1b) montre un envasement progressif de la zone côtière à partir de $25 \mathrm{~m}$ de profondeur. Celuici se situe non pas directement en face de l'embouchure du fleuve, mais légèrement au sud à cause de la circulation côtière principalement dirigée du nord vers le sud. Il met en communication la vasière médiane et le prodelta. 


\section{Matériel et méthode}

Le suivi des apports du fleuve Têt a été réalisé grâce à la mise en place d'une station de suivi automatique de ses apports liquides et solides à Canet-enRoussillon en amont de l'embouchure (Figure 1b). Les débits liquides sont mesurés à Perpignan en amont de l'embouchure par l'agence de l'eau de l'Aude.

L'impact des apports du fleuve Têt en mer est étudié à partir de la Plateforme d'Observation de l'Environnement Méditerranéen du Littoral LanguedocRoussillon (POEM-L2R) localisée à environ 2,5 km de l'embouchure du fleuve Têt sur son prodelta. Cette station météo-océanique abrite des instruments situés sur un fond de $28 \mathrm{~m}: 1$ altimètre acoustique mesurant la variabilité de la hauteur du niveau sédimentaire et un ADCP de fond, équipé d'un capteur de houle, mesurant les paramètres de la houle pendant 20 minutes toutes les 3 heures ainsi que le courant sur toute la colonne d'eau entre les périodes de mesure de vague. Les instruments ont été déployés en continu à partir d'octobre 2004 jusqu'à octobre 2005.

L'intensité de rétro-diffusion du signal acoustique de l'ADCP a également été analysée afin d'estimer les concentrations de matières en suspension (MES) dans la colonne d'eau. Ces concentrations sont calculées à partir de l'énergie acoustique transmise par l'ADCP, de la quantité de matière contenue dans la colonne d'eau et de l'atténuation du son dans l'eau.

\section{Résultats et discussion}

La période hivernale 2004-2005 a été une période relativement peu intense en terme d'évènements climatiques extrêmes contrairement à la période hivernale 2003-2004 ${ }^{[4]}$. On a cependant observé plusieurs évènements météo-marins qui ont eu un impact significatif au niveau sédimentaire sur le site étudié : des évènements de crue (décembre 2004), des évènements de tempête (avril 2005) et des évènements de tempête associés à des crues (février et novembre 2005). Ces derniers évènements sont les plus fréquents et les plus importants, et ont un impact majeur sur le transit sédimentaire côte-large.

\subsection{Les apports continentaux}

Le fleuve Têt a un débit moyen de $10 \mathrm{~m}^{3} / \mathrm{s}$, son débit minimum est de seulement quelques $\mathrm{m}^{3} / \mathrm{s}$ en période estivale, mais lors des brèves crues-éclair automnales, il peut atteindre $1800 \mathrm{~m}^{3} / \mathrm{s}$ en débit instantané avec une charge particulaire mesurée maximale de $47 \mathrm{~g} / 1$ pendant la crue de $1940{ }^{[3]}$. Durant la période étudiée, le fleuve Têt a seulement subi quelques crues mineures $\left(\mathrm{Qj}<100 \mathrm{~m}^{3} / \mathrm{s}\right)$ et une crue majeure le 15 novembre 2005 (Figure 2b) avec un débit instantané supérieur à $500 \mathrm{~m}^{3} / \mathrm{s}$ (période de retour de 2 à 5 ans). Cette crue a exporté environ 18000 tonnes de sédiment soit plus de $70 \%$ des apports solides annuels du fleuve Têt déchargés dans la zone littorale en seulement 3 jours. 


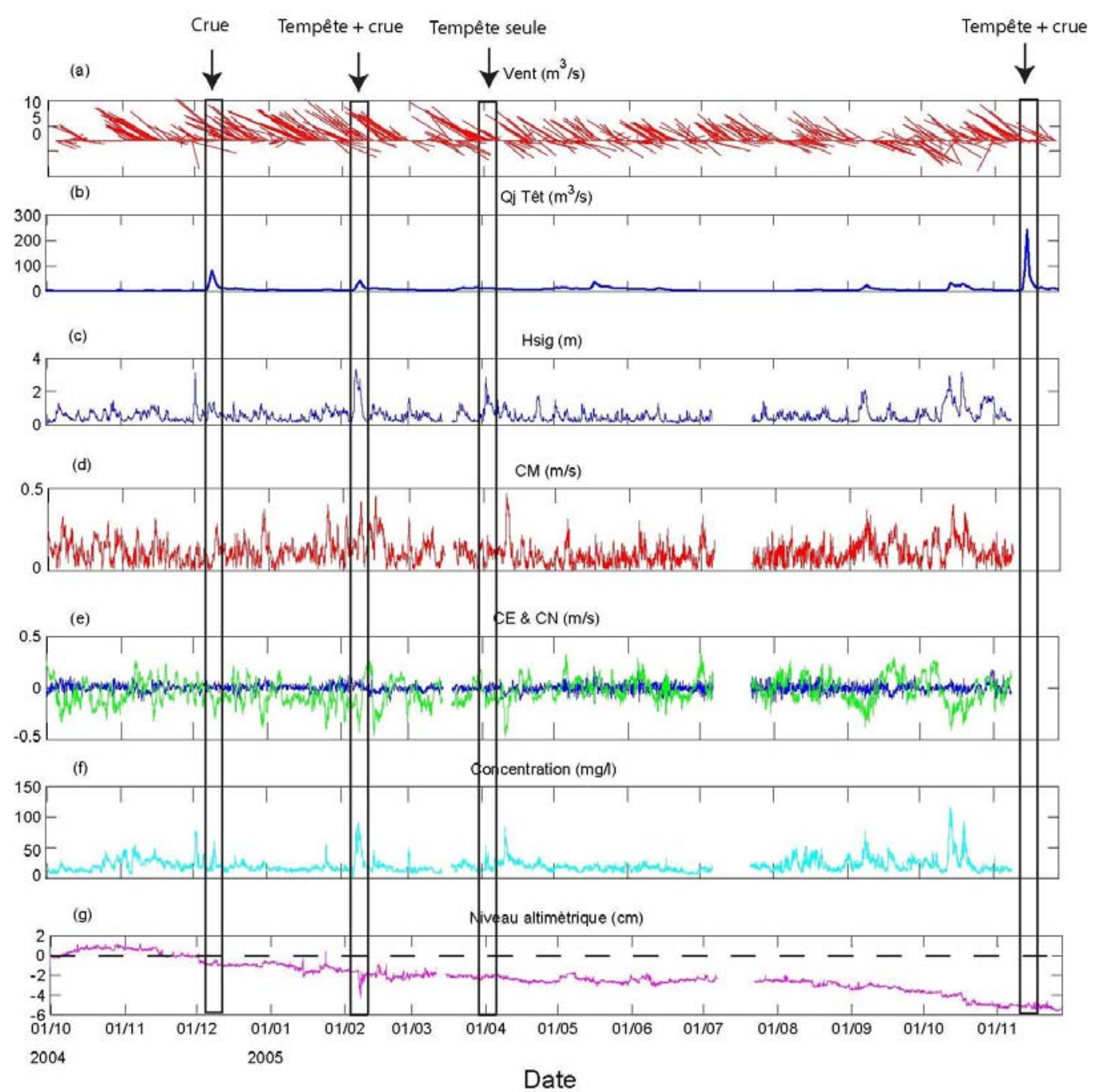

Figure 2: Séries temporelles de l'intensité et de la direction du vent (a), des débits journaliers de la Têt (b), de la hauteur significative des vagues (c), de l'intensité des courants de fond (d), des composantes est-ouest (en bleu) et nord-sud (en vert) du courant de fond (e), de la concentration particulaire (f) et du niveau altimétrique du fond (g) au niveau du prodelta de la Têt.

\subsection{Les tempêtes}

Le littoral roussillonnais est régulièrement soumis à l'influence de tempêtes de sud-est. La hauteur et la période moyennes significatives de cette houle sont de $0,6 \mathrm{~m}$ et 4,5 s (Figure 2c). Ces évènements sont généralement accompagnés par des vents marins de forte intensité (Figure 2a) et interviennent essentiellement durant la période hivernale. La tempête la plus forte mesurée pendant la période étudiée, s'est produite en février $2005(\mathrm{Hs}=3,31 \mathrm{~m}$ et $\mathrm{Tp}=8 \mathrm{~s})$ avec une période de retour annuelle. Ces évènements de tempête génèrent des courants de fond alternatifs capables de remobiliser les sédiments prodeltaïques qui sont ensuite transportés par les courants. Les vitesses orbitales maximales atteintes ont été mesurées lors de la tempête de février 2005 et atteignent $52 \mathrm{~cm} / \mathrm{s}$. Sachant, que des vitesses orbitales de $10 \mathrm{~cm} / \mathrm{s}$ sont suffisantes pour mettre en mouvement les 
particules sédimentaires du fond (D50 $=80 \mu \mathrm{m})$, ces vitesses sont atteintes $5 \% \mathrm{du}$ temps lors de la période étudiée essentiellement en hiver.

\subsection{Les courants}

L'intensité moyenne des courants à $2 \mathrm{~m}$ au dessus du fond est de $11 \mathrm{~cm} / \mathrm{s}$ sur l'année (Figure 2d). Si l'on considère l'intensité des courants de fond en terme de remise en suspension, ceux-ci excèdent une valeur critique de $10 \mathrm{~cm} / \mathrm{s} 50 \% \mathrm{du}$ temps soit la moitié de l'année. La composante longitudinale (moyenne $=32$ $\mathrm{cm} / \mathrm{s}$ ) est plus importante que la composante transversale (moyenne $=2 \mathrm{~cm} / \mathrm{s}$ ) du courant de fond (Figure 2e). La résultante sur une année de la composante transversale est pratiquement nulle, cependant les vitesses instantanées peuvent atteindrent parfois $20 \mathrm{~cm} / \mathrm{s}$. La composante longitudinale du courant, est plus intense en hiver avec des vitesses moyennes près du fond de près de $50 \mathrm{~cm} / \mathrm{s}$. Durant la période hivernale 2004-2005, les vents dominants ont été particulièrement intenses pendant plusieurs mois, ce qui a induit une circulation côtière stable à l'origine de forts courants dirigés vers le sud. En période estivale, ce même courant est beaucoup plus faible et est légèrement dirigé vers le sudouest.

\subsection{Les concentrations de matières en suspension près du fond et les variations du niveau du fond}

Les concentrations de MES mesurées près du fond ( $2 \mathrm{~m}$ au dessus du fond) varient de 2 à $115 \mathrm{mg} / \mathrm{l}$ durant la période étudiée (Figure 2, f). Etant donné qu'il n'y a pas eu d'apports solides par le fleuve Têt, l'essentiel des concentrations de MES mesurées proviennent de la remise en suspension par les houles de sud-est. En effet les plus fortes concentrations sont généralement observées lors des évènements de tempête (décembre 2004, février 2005 et octobre 2005). Mais, on observe également des pics de turbidité comme en avril 2005 qui sont reliés à de forts courants de fond générés par le vent.

Durant la période d'octobre 2004 à octobre 2005, le niveau altimétrique du fond a baissé de près de $6 \mathrm{~cm}$. On n'a pas observé de baisse brusque du niveau des sédiments lors des évènements de tempête, mais plutôt une baisse lente et progressive de celui-ci tout au long de la période étudiée, en relation avec de forts et constants courants de fond également induits par le vent.

\subsection{Le transit sédimentaire}

Les flux de matières en suspension mesurés au niveau de la bouée POEM sur un fond de $28 \mathrm{~m}$ indiquent une très forte composante longitudinale et une faible composante transversale à $2 \mathrm{~m}$ au dessus du fond(Figure 3a). Evidement l'essentiel du transport sédimentaire se produit dans les premiers $\mathrm{cm}$ près du fond, mais ces mesures nous donnent une indication précieuse sur le sens et l'intensité $\mathrm{du}$ transport particulaire sur ce type de prodelta (Figure $3 \mathrm{~b}$ ). On observe une première période d'octobre 2004 à avril 2005 où les flux particulaires longitudinaux à la côte sont relativement importants correspondant à la période hivernale des tempêtes. Ensuite, on observe une période plus calme en terme de flux, qui correspond à la période estivale. Puis on observe une reprise du transport particulaire vers le sud, sud-est avec les premières tempêtes hivernales de 2005. 

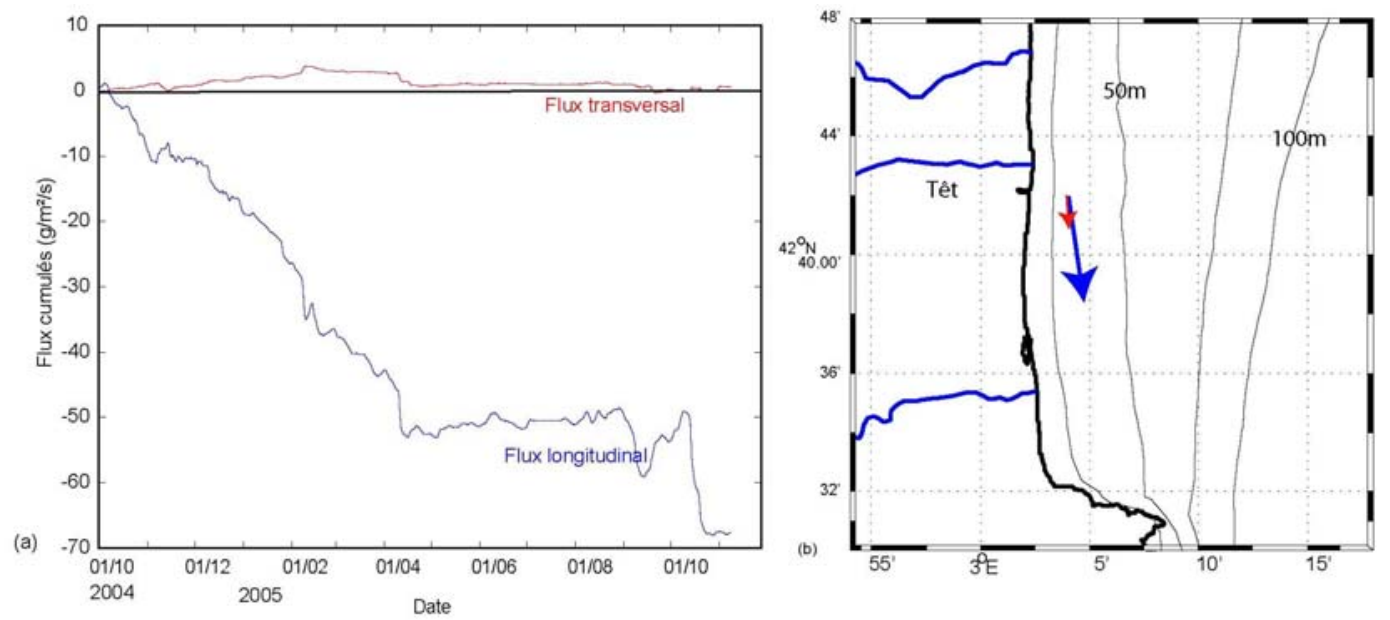

Figure 3 : (a) Flux cumulés de matières en suspension estimées à partir des mesures acoustiques de l'ADCP de fond sur le site POEM à $28 \mathrm{~m}$ de profondeur dans les directions nord-sud (ligne bleue) et est-ouest (ligne rouge), et (b) résultantes du transit des matières en suspension en hiver (flèche bleue) et en été (flèche rouge).

Le prodelta de la Têt, caractéristique des petits prodeltas méditerranéens soumis à des apports continentaux discontinus et à l'action des tempêtes, semble agir comme une zone de "bypass » sédimentaire. Le matériel injecté principalement lors des évènements de crue se dépose temporairement dans la zone prodeltaïque, et est ensuite resuspendu lors des évènements de tempête. Le matériel ainsi resuspendu est advecté par les courants principalement vers le sud-est et alimenterait la vasière médiane située vers $50 \mathrm{~m}$ de profondeur [9]. Seul le prodelta du Rhône, avec des apports continus de matériel continental possède un taux d'accumulation significatif et enregistre un signal sédimentaire net [6]. La tendance sédimentaire actuelle des petits prodeltas méditerranéens serait la formation d'une couche superficielle continuellement remaniée et lessivée de sa fraction fine riche en matière organique lors des évènements successifs de tempête. Cette tendance est d'autant plus accentuée que les apports continentaux sont affaiblis par la construction de barrages au niveau des bassins versants et que le nombre de tempêtes exceptionnelles semble augmenter en raison du changement climatique.

\section{Conclusion}

Cette étude nous a permis de montrer les principaux résultats suivants. En général, les vents continentaux dominants induisent une circulation littorale uniformément dirigée vers le sud-est. Les courants alternatifs générés par les houles de sud-est représentent les mécanismes principaux de remise en suspension du matériel fin dans la zone interne du plateau continental. Le seuil de remise en suspension par ce type de courants n'est atteint que $5 \%$ du temps essentiellement en période hivernale avec des vitesses orbitales supérieures à $50 \mathrm{~cm} / \mathrm{s}$. Cependant, l'intensité des courants près du fond a été particulièrement forte sur la période hivernale étudiée à cause des vents continentaux soutenus, et la vitesse des courants a été supérieure au seuil de remise en suspension (estimées à $10 \mathrm{~cm} / \mathrm{s}$ ) $50 \%$ du temps 
de l'année. Durant la période étudiée, il n'y a pas eu de trace d'un quelconque dépôt sédimentaire en relation avec les apports fluviaux. Mais, les mesures altimétriques indiquent une érosion lente et continue du fond sédimentaire $d u$ prodelta, de l'ordre de $6 \mathrm{~cm}$ sur un an. Les flux de matières en suspension montrent une forte composante longitudinale vers le sud et une faible composante transversale vers l'est. Le matériel resuspendu au niveau de la zone prodeltaïque est advecté par les courants vers le sud-est et alimenterait la vasière située au milieu du plateau continental. Les prodeltas joueraient alors le rôle de zone de dépôt temporaire du matériel injecté par les fleuves côtiers avant d'être resuspendu et dirigé vers le large par la circulation moyenne du courant sur le plateau. Alors que les sédiments grossiers littoraux suivent la dérive littorale du sud vers le nord, les sédiments fins prodeltaïques sont dirigés dans le sens contraire. Les prodeltas agissent ainsi comme des réacteurs sédimentaires et serviraient à alimenter les zones vaseuses plus profondes avant d'être exporté hors du plateau continental.

\section{Bibliographie}

1 Aloisi J.-C., Monaco A., (1975). La sédimentation infralittorale : les prodeltas nord-méditerranéens. C.R. Acad. Sci. Paris, 280, série D, 2833-2836.

2 Roussiez V., Aloisi J.-C., Monaco A. and Ludwig W., (2005). Early muddy deposits along the Gulf of Lions shoreline: A key for a better understanding of land-to-sea transfer of sediments and associated pollutant fluxes. Marine Geology, 222-223, 345-358.

3 Serrat P., Wolfgang L., B. Navarro et J.-L. Blazi, (2001). Variabilité spatiotemporelle des flux de matières en suspension d'un fleuve côtier méditerranéen : la Têt (France). Comptes Rendus de l'Académie des Sciences, 333, 389-397.

4 Guillén, J., Bourrin, F., Palanques, A., Durrieu de Madron, X., Puig, P. and Buscail, R. Sediment dynamics during wet and dry storm events on the Têt inner shelf (SW Gulf of Lions). Marine Geology (sous presse).

5 Certain R., (2002). Morphodynamique d'une côte sableuse microtidale à barres : le Golfe du Lion (Languedoc-Roussillon). Thèse de 3ème cycle, Université de Perpignan, $209 \mathrm{p}$.

6 Ulses C., (2005). Dynamique océanique et transport de la matière particulaire dans le Golfe du Lion : Crue, tempête et période hivernale. Thèse de 3eme cycle, Université Paul Sabatier, Toulouse, 247 p.

7 Monaco A. et Aloisi J.C., (2000). Carte de la nature des fonds du golfe du Lion. Site ORME (http://medias.obs-mip.fr/orme/).

8 Radakovitch, O., Charmasson, S., Arnaud, M. and Bouisset, P., (1999). 210Pb and caesium accumulation in the Rhône delta sediments. Estuarine Coastal and shelf Sciences, 48, 77-92. 